Running head: COUPLE FUNCTIONING AFTER PEDIATRIC CANCER

Couple Functioning after Pediatric Cancer Diagnosis: A Systematic Review

\author{
Marieke Van Schoors, MSc \\ Department of Experimental Clinical and Health Psychology, \\ Ghent University, Ghent, Belgium \\ Line Caes, Ph.D. \\ School of Psychology and Centre for Pain Research, \\ National University of Ireland Galway, Galway, Ireland \\ Melissa A. Alderfer, Ph.D. \\ Center for Healthcare Delivery Science, \\ Nemours Children's Health System, Wilmington, DE and \\ Department of Pediatrics, Sidney Kimmel Medical College \\ Thomas Jefferson University, Philadelphia, PA \\ Liesbet Goubert, Ph.D. and Lesley Verhofstadt, Ph.D. \\ Department of Experimental Clinical and Health Psychology, \\ Ghent University, Ghent, Belgium
}

Address correspondence to:

Marieke Van Schoors, Department of Experimental Clinical and Health Psychology, Ghent University, H. Dunantlaan 2, B-9000 Ghent, Belgium

Tel: $+32-9-264.86 .11$ - Fax: $+32-9-264.64 .89$.

E-mail: marieke.vanschoors@ugent.be 
Note: This is an uncorrected version of an author's manuscript accepted for publication. Copyediting, typesetting, and review of the resulting proofs will be undertaken on this manuscript before final publication. During production and pre-press, errors may be discovered that could affect the content. 


\begin{abstract}
Objectives: A systematic review was conducted to: 1) investigate couple functioning after a pediatric cancer diagnosis, and 2) examine theoretical and methodological tendencies and issues in this literature.

Methods: Searches of Web of Science, Pubmed, Cochrane, PsycInfo and Embase resulted in inclusion of 32 qualitative, quantitative or mixed method papers. Findings of these papers were extracted for summary.

Results: Most couples adapt well to the crisis of a pediatric cancer diagnosis in domains such as emotional closeness, support, marital satisfaction and general marital adjustment. However, most experience difficulties in the domain of sexual intimacy and reports on conflict are mixed across qualitative and quantitative studies.
\end{abstract}

Conclusions: This review illustrates the need for future research with a greater focus on the impact of a pediatric cancer diagnosis on the couple's functioning, conducted with use of appropriate theoretical frameworks and based on both partners' reports. Improvements in research are needed to best inform couple-based interventions.

Keywords: pediatric cancer; oncology; couples; intimate relationships; parents; systematic review 


\section{Couple Functioning after Pediatric Cancer Diagnosis: A Systematic Review}

Due to advances in treatment, approximately $76 \%$ of children diagnosed with cancer survive [1], many with long-term and late effects. Thus, pediatric cancer is now considered a chronic illness. Like all chronic illnesses, pediatric cancer not only impacts the diagnosed child [2], but all family members $[3,4]$. Family members and the family system as a whole need to adapt to the unpredictable and uncontrollable course of cancer and its treatment [5].

Research has been accumulating focused upon individual adaptation of patients [6], parents $[7,8]$ and siblings $[3,9]$. There have also been recent efforts at summarizing the literature on the adaptation of the family system as a whole after pediatric cancer diagnosis [10], providing evidence that most families return to, sustain, or achieve adaptive levels of family functioning after this challenge. However, research into the adaptation of family subsystems is less common. Subsystems within the family are relational units marked by invisible interpersonal boundaries based upon specific characteristics (e.g., age or generation) or function. Subsystems within the family often have different relationship rules and patterns of interaction [11]. For example, children within the family form one subsystem, parents form a subsystem in their role of providing and caring for the children within the family, whereas the adult couple form yet another subsystem. In particular, little is known about how the couple subsystem -more specifically the intimate relationship of the diagnosed child's parents- is affected by pediatric cancer. This apparent gap in the research literature is somewhat surprising given that three related but distinct areas within the family psychology literature (i.e., social ecology, stress and coping, intimate relationship science) point toward the likelihood that the couple subsystem will be impacted by childhood cancer. First, the Social Ecology Model [12] postulates that an individual is embedded in a broad social context, and that a stressor (like pediatric cancer) will influence the 
development and adaptation of the individual (i.e., the child with cancer) as well as the context in which this individual lives and the subsystems with which s/he interacts, including the couple subsystem. Second, family stress and coping models (e.g., Double ABCX-model [13]; FAARmodel [14]), specify pathways through which external stressors impact family systems and their subsystems, including the couple subsystem (see Bradbury \& Karney [15]). Third, most theories on how intimate relationships succeed or fail focus upon the powerful role that circumstances outside the relationship can play in shaping experiences within the relationship [15]. Taken together, within the broader family psychology literature, the relationship between married or cohabiting partners has become one of the most frequently studied and measured components of the family system [16] and has been considered to be the actual core element of the family system [17].

Engagement in a wide range of coping and coping assistance strategies is reported by parents of a child with cancer [18]. Consequently, it is plausible to assume that the stressors accompanying pediatric cancer and its treatment require both material and emotional resources (e.g., time, emotional availability) from the child's parents, which then cannot be invested in the maintenance of their intimate relationship. Moreover, the stressors may also give rise to conflict within the couple. Across research studies, findings reveal both reduced and enhanced levels of relationship quality after pediatric cancer [19]. However, to date no attempts have been made to provide a systematic and critical integration of the available evidence. As such, a systematic review addressing the couple subsystem in the context of pediatric oncology would add substantial value to our understanding of how couples adapt to pediatric cancer diagnosis. The primary aim of this review was to investigate the impact of a pediatric cancer diagnosis on couple functioning. A secondary aim was to examine theoretical and methodological patterns and issues in the literature and to formulate recommendations for future research and clinical practice. 
Method

The current review is the third in a series of systematic reviews summarizing qualitative and quantitative evidence of family and couple functioning after a pediatric cancer diagnosis [20, 21]. While the previously published reviews focused on family-related and individual child functioning in the context of pediatric cancer, the focus of the current review is on relationship functioning within the couple subsystem. All reviews followed a strict scientific method, as outlined by Eiser, Hill and Vance [22] and the Cochrane Collaboration [23], to conduct a rigorous systematic search and provide a reliable and unbiased overview of the findings (see Van Schoors et al. ${ }^{20}$ for more details). A literature search was conducted in July 2014 and was updated in October 2015 to include the most recent published articles on this topic.

Literature Search and Inclusion Criteria

Web of Science, Pubmed, PsycInfo, Cochrane and Embase were searched using the following search terms: (cancer OR tumor OR malignancy OR oncolog*), (child* OR pediatric), (family OR parental OR marital OR marriage OR sexuality OR couple) and (psycholog* OR adaptation OR adjustment). Studies were retained if the article: a) examined the impact of pediatric cancer diagnosis (0 -18 years; any type of cancer) and treatment on any aspect of couple functioning; b) was written in English; c) presented new, empirical qualitative or quantitative data (i.e., reviews, case reports, commentaries, books, practice guidelines, conference abstracts and dissertations were excluded); and, d) did not exclusively focus on palliative care or bereavement, as these experiences are distinct from general adjustment to pediatric cancer and may have a different impact upon couple functioning. Studies focusing on both curative and palliative care were retained. No restrictions were placed on studies related to publication date. Study Selection 
The original search, in July 2014, identified a total of 5660 papers, which were independently screened by the first and second author in three separate steps: a) title screening (100\% screened by the first and second author; 1,592 articles retained with $89 \%$ agreement); b) abstract screening (100\% screened by the first and second author; 427 articles retained with $83 \%$ agreement); and, c) full text screening (100\% screened by the first author, $25 \%$ by the second author; $87 \%$ agreement). This 3-step selection process resulted in a total of 29 articles retained for the purpose of this review. Across our 3-step selection process, for 191 articles (although judged as not relevant based upon title or abstract) no full text was available. Disagreements between screeners were discussed and resolved; where necessary a third reviewer was consulted. In addition, the reference lists of the retained articles were scanned to identify additional relevant articles, resulting in the addition of two papers. Lastly, to ensure up-to-date results, the search was repeated in October 2015 and 449 new potentially relevant papers were identified. The same 3-step screening process was followed for this updated search: 68 were retained based on their title (87\% agreement between first and second author); 17 were retained based on abstract (75\% agreement between first and second author); and 1 was retained after full text screening. In sum, the current review is based on a final set of 32 articles (see Figure 1).

\section{Data Extraction}

The main purpose of this systematic review was to provide a narrative synthesis of the current state of knowledge on couple functioning after childhood cancer diagnosis. This was done by adopting a textual approach with evaluation of the scientific merit of the available evidence. A data abstraction sheet was developed to ensure systematic and standardized data extraction (available upon request). The data abstraction sheet identified the following study aspects: a) study characteristics such as year of publication, journal and database; b) which, if any, theoretical framework was used; c) methodological and statistical aspects, such as design (e.g., 
cross-sectional versus longitudinal), sample size, unit of measurement, and assessment of interdependence (i.e., appropriately accounting for the interdependence of partner reports within couples); and, d) a summary of the general findings. The first author conducted the data extraction for all the included articles. To ensure accuracy, the second author conducted data extraction for $25 \%$ of the articles (i.e., full text screening; $87 \%$ agreement).

A meta-analysis of the gathered data was deemed inappropriate as we preferred to include qualitative findings to ensure a comprehensive review. Furthermore, with regard to the quantitative studies, either too few studies were identified assessing a particular construct to warrant a meta-analysis (i.e., less than 2 studies identified for the same construct) or too much heterogeneity was observed in sample characteristics and outcomes.

In addition, each included study was rated by the second author with respect to its scientific merit using the criteria published in Alderfer and colleagues (2010). Quantitative studies were evaluated for explicit scientific purpose, appropriateness of design and analysis, measurement reliability, statistical power and approach, internal and external validity, appropriate discussion, and knowledge contribution. Qualitative studies were evaluated for explicit scientific purpose, appropriateness of design and analysis, grounding results in examples, integration of results into a framework, specification of author's perspective, accurate and understandable topic coverage, application of credibility checks, and appropriateness and description of sample [3]. Each aspect was rated on a 3-point scale ranging from $1=$ "no or little evidence in fulfilling the criterion or low quality" to 3 = "good evidence or high quality". An overall score for scientific merit was obtained for each study by averaging the individual aspect scores. Reliability of the quality assessment was assured by double coding of $33 \%$ of the included studies by the first author. This revealed excellent interrater reliability as evidenced by a single measure and average measures intraclass correlation coefficients across the two raters of .92 and .96 , respectively. 


\section{Results}

Part I: Characteristics of Reviewed Studies

The methods and findings of the 32 reviewed studies are summarized in Supplemental Table 1. About half of the reviewed studies were quantitative $(n=17,53 \%)$. Thirteen studies $(41 \%)$ used qualitative methods; two used mixed methods designs (6\%). About two-thirds of the studies were cross-sectional $(n=21)$ and the rest were longitudinal $(n=11)$. Sample size ranged from 8 individual partners to 328 partners/164 couples. In $35 \%$ of the studies, only one partner participated (female partner: $n=4$ studies; male partner: $n=2$ studies, gender not specified: $n=$ 8 studies). In the other studies, couples ( $n=8$ studies) or a combination of couples and individual partners ( $n=10$ studies) were included. Ten studies (31\%) only included married couples, whereas 17 studies (53\%) used a mix of married, cohabiting, or other couples (i.e., divorced parents, single parents, step-families, remarried parents and widowed parents). In five studies (16\%), marital status was not reported. A wide variety of cancer diagnoses were included, with leukemia, lymphoma and brain tumors as the most frequently represented. Time since diagnose ranged from new diagnoses to 7 years post-diagnosis, but was not reported in two studies and vague in seven others ("long term survivors", "in treatment').

Part II: Narrative Summary of Reviewed Studies

Seven distinct dimensions of couple functioning emerged from the research literature: emotional closeness, marital conflict, marital support, communication, sexual intimacy, marital satisfaction and general marital adjustment. Within each of the following subsections, a brief explanation of the dimension is given, followed by the number and type of included studies identified and a narrative summary of the findings across studies. Qualitative results are presented before quantitative results. 
Emotional Closeness. Emotional closeness refers to the feeling of positive connectedness between partners, varying from acquaintanceship to complete absorption of self and other into oneness [24]. This dimension was investigated in five qualitative studies, one quantitative study and one mixed method study.

Across four qualitative studies, all including samples of families both on- and offtreatment, participants often indicated that couple connectedness was strengthened by the illness [25-28]. In two studies involving on-treatment families, however, a range of responses was revealed. One of these studies reported that $60 \%$ of the participants $(n=23)$ indicated an increase in couple connectedness since diagnosis, $34 \%(n=13)$ reported no change and $5 \%(n=2)$ reported a decrease in connectedness $\left[29^{\mathrm{mix}}\right]$. In the second study, $45 \%$ of the participants $(n=$ 32) reported an increase, $17 \%(n=12)$ reported no change and 38\% $(n=27)$ reported a decrease in emotional closeness [29]. Variability in the experience of closeness has been proposed to reflect baseline differences across families [29] or differences in the illness and treatment challenges faced by couples [27]. The one quantitative study of couple's closeness indicated that female partners reported a significant increase in emotional closeness with their partners from diagnosis to one year-post diagnosis [30].

In summary, most studies provided evidence for increased emotional closeness within the couple after diagnosis of pediatric cancer. However, this pattern clearly does not characterize all couples. More research into which couples draw closer and which do not is needed. Closeness before diagnosis and disease/treatment characteristics might play an important role.

Marital Conflict. Marital conflict refers to overt opposition or disagreement between partners that is identified as a source of difficulty in the relationship [31]. This dimension was addressed in five qualitative studies and three quantitative studies. 
Across the qualitative studies, subsets of participants reported increased conflict during treatment $[29,32]$, after treatment $[19,33]$ and across the illness trajectory [28]. However, an increase in conflict frequency was not reported by all couples in these studies: some reported no changes, whereas others indicated having fewer arguments, as they channeled all their energy into caring for the sick child and had no time to argue [32].

In included quantitative work, one study compared the yearly divorce rate of families with pediatric cancer with the rate in the general population and found no significant differences [34]. The second quantitative study indicated that only $8 \%$ of female participants $(n=2)$ and $5 \%$ of male participants $(n=1)$ experienced regular difficulties with their partner within three months after diagnosis. Nine months later, $21 \%$ of the female participants $(n=4)$ and $7 \%$ of the male participants $(n=1)$ reported regular difficulties, a non-significant difference across time [35]. In the third quantitative study, some couples reported an improvement in conflict resolution skills after their child's cancer diagnosis [36].

In summary, reports of conflict were found in samples consisting of both on- and offtreatment families. However, the question remains whether this frequency of conflict transcends the frequency of conflict inherent to all couples. Sample characteristics (e.g., diagnosis, age of child; country of origin) and aspects of study design (e.g., measure, sample size) did not seem to be associated with findings regarding conflict.

Marital Support. Marital support refers to assistance, encouragement and caring provided by one partner and received or perceived by the other [37]. This dimension was addressed in nine qualitative studies, two quantitative studies and one mixed method study.

Across the included qualitative studies, the partner was described as a highly important source of support $[25,27,29,32,33,36,38,39]$ and sometimes even the most relied upon source of support [40], both during and after treatment. Participants turned to their partners for all kinds 
of support, including emotional (e.g., listening to each other) and practical (e.g., maintaining the home) support $[29,36]$. However, within these same studies, some individual participants reported that their partners were so impacted by the diagnosis or so focused on the ill child that they could not provide adequate partner support [29, 32, 36, 39]. For example, in one study, $14 \%$ of the participants $(n=9)$ described their partner as not at all supportive during treatment [ $38^{\text {mix }}$.

The first included quantitative study investigating partner support indicated that the spouse was one of the most frequently used sources of support and the most helpful source of support [41]. The second study indicated that perceived partner support was consistent across time from diagnosis until 1 year post-diagnosis (i.e., at diagnosis, 6 months and 12 months later; [42]). These results are consistent with the qualitative studies.

In summary, across qualitative and quantitative studies, most partners reported that their partner's support was important, available to them, and helped them cope with the cancer experience. However, not every partner was equally able to provide such support, sometimes resulting in unmet support needs.

Communication. Communication is the interchange of thoughts, feelings, experiences and information within the couple [43]. This dimension was investigated in three quantitative studies.

The first quantitative study addressed couple agreement in reports of communication style at diagnosis and demonstrated that male and female reports did not differ in the amount of perceived avoidance (e.g., withdrawal, avoiding conflict, silence), incongruent (e.g., dishonesty or preference for talking to others) and destructive (e.g., insulting, irritated or abusive) communication. However, female partners did experience less mutual understanding and sharing in their relationship than male partners [44]. According to the second study, only presenting data of couples with inter-spousal agreement on communication patterns, $29 \%$ of the couples $(n=10)$ reported positive changes since the cancer diagnosis, 20\% $(n=7)$ reported no changes and none 
reported negative changes [36]. In a third study, mothers of children undergoing hematopoietic stem cell transplantation (HSCT) reported equal amounts of partner criticism and avoidance during the first six months after HSCT, with no significant changes over time [45].

In summary, most available studies focused on comparison between partner reports on communication, revealing few differences. However, the evidence regarding changes in communication patterns between partners after a child's cancer diagnosis is too sparse to draw strong conclusions. There have been no comparisons between communication patterns among couples with children with cancer and couples with healthy children and no qualitative reports.

Sexual Intimacy. Sexual intimacy involves physical closeness between partners [46]. This dimension was investigated in three qualitative studies and one quantitative study.

All three qualitative studies indicated that the pediatric cancer diagnosis had a negative impact on the sexual relationship of the participants [19, 27, 47]. More specifically, participants indicated that intimacy had "gone out the window" [27, 47], as there was no privacy or time to spend together due to the constant attention and care needed by their child [47]. Of note, one study found that worry -not lack of love- was the reason that couples reported being too drained for sex during their child's illness trajectory [19]. The one included quantitative study revealed that in nearly half of the couples both partners reported a deterioration in their sexual relationship. In this same study, only one couple reported improvements [34].

In summary, across qualitative and quantitative studies, couples reported that the cancer diagnosis negatively affected their level of physical intimacy and sexuality. The magnitude of this effect and full understanding of the underlying reasons, however, remain elusive.

Marital satisfaction. Marital satisfaction refers to partners' global sentiment or happiness with their relationship [48]. This dimension was investigated in six quantitative studies and one mixed method study. 
Across studies, a decrease in marital satisfaction was reported during the first year after diagnosis by both male [49] and female partners [44, 49], with the highest level of dissatisfaction during the first two months after diagnosis [50] and significantly higher levels of satisfaction after treatment completion (i.e., 2 year after diagnosis; [51]. When compared with population based control groups, no significant differences were found during $[35,44]$ or after treatment $[44$, 49]. At diagnosis, levels of marital satisfaction were lower than those of well-adjusted couples in the general population, but higher than couples referred for couples therapy [52]. At one year post-diagnosis, marital satisfaction was also higher for those couples with a child with cancer compared to couples referred for therapy [49]. Surprisingly, parents of children who had relapsed reported levels of marital satisfaction similar to those of parents of newly diagnosed children [50], as well as those of parents of children who had survived [44].

Some inconsistencies emerged across studies when examining gender differences in marital satisfaction. Two studies, both involving a mix of on- and off-treatment families, found no differences between male and female participants regarding their reported level of marital satisfaction [44, 49]. One study [50] conducted in Taiwan, found that female partners were more dissatisfied than male partners. A third study $\left[53^{\mathrm{mix}}\right.$ ] from the United States conducted repeated measures analyses separately for males and females, then compared results and found that male partners were more dissatisfied than female partners.

In summary, a U-shaped curve for marital satisfaction emerged over time, with a decrease in marital satisfaction in the first year after diagnosis and a gradual increase across later stages of the illness trajectory. In addition, participants reported levels of marital satisfaction that were comparable to population based control groups; lower than well-adjusted couples and higher than samples with recognized marital problems. Gender differences in marital satisfaction remained unclear, although culture may play a role. 
General marital adjustment. General marital adjustment refers to a broad scope of outcomes, including a consideration of marital processes and marital outcomes [48]. While general marital adjustment overlaps with all above-addressed outcomes, the studies summarized in this section assessed marital adjustment as a general construct without providing details on different specific dimensions of marital functioning. This general construct was addressed in three qualitative studies, seven quantitative studies and one mixed method study.

Two qualitative studies investigated the impact of childhood cancer survival on general marital adjustment, revealing that only a minority reported the marital relationship was in jeopardy as a consequence of the diagnosis [54]. While equal reports of positive change (e.g., "This even made our marriage stronger", $23 \%$ of the participants $(n=21))$ and marital difficulties (25\% of participants $(n=24))$ emerge, among those reporting difficulties, $46 \%(n=11)$ remained married, 33\% $(n=8)$ reported divorce as a direct result of the child's illness, and 21\% $(n=5)$ reported divorced due to problems prior to the illness. The last qualitative studies addressed similarities in perception between male and female partners, and found that $79 \%$ of spouses ( $n=$ 23) agreed regarding the impact of cancer on the quality of their marital relationship [29].

The quantitative studies indicated that only a minority of couples reported that pediatric cancer diagnosis had a major negative impact on their marital adjustment and hardly ever resulted in divorce [55]. In one study, $72 \%$ of the participants $(n=46)$ reported that the marital relationship presented no problem during treatment and only $5 \%(n=2)$ indicated marital adjustment to be a major concern $\left[38^{\mathrm{mix}}\right]$.

Similar reports of marital distress were observed across gender with $25 \%$ of female partners $(n=17)$ and $28 \%$ of male partners $(n=19)$ reporting clinically elevated levels of marital distress during the first weeks after diagnosis [56]. Twenty months later, these numbers were 19\% $(n=8)$ and 24\% $(n=10)$ [57], respectively. However, inconsistencies emerged regarding 
differences in reports of marital quality as a function of gender. While Barbarin and colleagues [38], Cornman [58] and Lavee [59] did not find any differences between male and female partners' reports of (changes in) marital quality, Lavee and Mey-Dan [34] found that men tended to perceive more positive changes than their female partners.

Inconsistencies also emerged regarding changes over time in marital quality. While one study reported an absence of significant change across time [57], others reported either curvilinear change (i.e., a deterioration during the first year followed by improvements across years two and three post-diagnosis; [34]) or both positive or negative change across the illness trajectory [59].

When marital adjustment during cancer treatment was compared with population based norms or control groups, parents of children with cancer were found to be similar to parents of healthy children [60], but less well-adjusted than the norms of married American couples [55, 58]. Parents of children with cancer, however, have been consistently found to be better adjusted than divorced couples [58] or couples experiencing marital problems [55].

In summary, research indicates that childhood cancer has the potential to negatively affect marital adjustment; however, for most couples their marital adjustment, even in this time of stress, is within normal limits and similar to controls. More research is needed to unravel the reasons for and impact of different reports across gender and time.

Part III: Evaluation of Reviewed Studies

On average the scientific merit of the included studies was good $(M=2.31$, range $=1.67$ to 2.78) with five studies scoring below 2.0 on a 3-point scale [3]. The most common weaknesses across studies were related to power (e.g., small sample size), failing to integrate the findings within a theoretical framework, internal validity (e.g., predominantly cross-sectional designs) and external validity (e.g., limited detail on saturation techniques and non-responders limiting 
generalizability). Areas of strength included well-justified objectives, selection of appropriate research methods and providing example quotes.

Theoretical considerations. In the majority of the studies $(n=25,78 \%)$, no theoretical framework was specified as guiding the research questions or selection of the variables. In the remaining studies with an underlying theoretical framework $(n=7,22 \%)$, however, it should be noted that none of the models used (i.e., ABCX-model, Family Empowerment model, TimeBound Model, Pediatric Medical Traumatic Stress Model, Family Stress Theory, FAAR-model) were specifically developed to understand how an external stressor like pediatric cancer diagnosis affects couples and their functioning. Instead, the models used in the reviewed studies were general stress and coping models, describing the general impact of a stressful situation on an individual and the entirety of the family context and subsystems with which s/he interacts, including the couple subsystem.

Measurement considerations. Even though the included studies focused upon marital constructs, only 18 studies (56\%) assessed couple functioning from the perspective of both partners. Eight of those studies only included data from couples (i.e., reports of both partners), whereas 10 studies included data from couples and individual partners. Studies including reports of both partners almost never analyzed the dyad [61], but rather did separate analyses for male and female partners. Because the unit of interest should harmonize with the unit of measurement [59], one could argue whether the reviewed studies adequately assessed couple functioning. Indeed, discrepancies in perceptions across family members (e.g., [63, 64] and couple members [15] speak to the need to collect data from both male and female partners, as well as to take the interplay of both partners' reports into account in statistical analyses.

Overall quality. As noted in our previous work [20,21], certain characteristics of the existing research base make it particularly difficult to draw strong conclusions. For instance, 
heterogeneity across and within studies with regard to sample characteristics and different operationalizations of marital constructs pose barriers to conduct meaningful meta-analysis. The reviewed studies tended to have small heterogeneous samples and to rely upon cross-sectional designs, precluding identification of factors that may reliably predict which couples experience the greatest difficulties after being confronted with the challenges posed by pediatric cancer. Further limitations of the reviewed studies include: a) most studies only described the experience of partners using qualitative quotes; b) only seven studies (22\%) used adequately demographically-matched control groups or norms; and c) only nine studies (28\%) statistically assessed changes in couple functioning over time. Furthermore, all but two studies exclusively relied on participant's self-reports of couple functioning, despite the known drawbacks associated with this method (e.g., social desirability), especially when dyadic processes (e.g., communication, supportive exchanges, and conflict) are under investigation [65].

\section{Discussion}

The results of this systematic review generally indicate that most couples adapt well after being challenged by pediatric cancer in domains such as emotional closeness, marital support, marital satisfaction and general marital adjustment. Thus, resilience, defined as a return to, sustainment, or achievement of competent levels of functioning after being confronted with a stressor such as pediatric cancer [66], seems to characterize couples with children with cancer across most of the identified couple constructs. However, conflict and lack of sexual intimacy may occur for some of these couples too.

These conclusions, however, need to be considered in the context of the following precautions. First, data regarding the functioning of the couple prior to the illness, longitudinal data examining changes in couple functioning over time since diagnosis, as well as criteria for judging whether the functioning of the couple is "adaptive" were rarely available. Instead, we 
frequently relied on qualitative quotes of partners regarding perceived changes in the couple's functioning. While comparisons with healthy controls or norms were sometimes available, it is unknown whether competent (couple) functioning in the context of pediatric cancer is the same as typical (couple) functioning within families of healthy children [10, 67].

Second, strong conclusions are also hampered by the relative lack of studies using a theoretical framework. In addition, when theoretical frameworks were used, they were not specifically tailored to the couple coping with stress. As a consequence, there was a lack of clarity in the differences and similarities between the marital concepts used in the reviewed studies. For example, marital satisfaction, marital quality and marital adjustment were used interchangeably in the reviewed studies, although theoretical frameworks and measurement guidelines for couple researcher encourage clear distinctions between the three constructs [48]. More specifically, marital satisfaction refers to "global marital sentiment or happiness as a unitary construct”; marital quality refers to "marital processes, such as quality of a couple's conflict management skills, supportive transactions, sexual relations, or emotional intimacy" and marital adjustment can be defined as " a consideration of marital processes such as conflict management skills and marital outcomes such as marital satisfaction”" ([48], p. 1028-1030).

Finally, most included studies utilized small, heterogeneous samples, and few studies are available within each dimension of couple functioning (ranging from 3 to 12 studies per dimension) thereby precluding robust conclusions. One possible reason for the limited amount of empirical work on the impact of a pediatric cancer diagnosis on couple functioning might be the lack of differentiation between partnership and parenthood. Indeed, in the context of pediatric cancer (both in research and clinical practice), partners are often addressed in their parenting role, which is also reflected in the large number of studies on parenting and pediatric cancer. However, the differentiation between partnership and parenthood is important, as both imply different 
responsibilities, roles, and behaviors [68, 69]. Moreover, men and women need to divide their energy and time between parenthood and partnership, as children (i.e., parenthood) make demands on couples that take time away from activities that promote and maintain their couple relationship (i.e., partnership), thereby pointing at the mutual influence between both $[15,70]$. Suggestions for Future Research

Future work, particularly studies adopting narrative techniques, should ideally rely on theoretical frameworks that incorporate partners' individual strengths and vulnerabilities (e.g., personality, family of origin experiences), external stressful events (e.g., low socio-economic status, previous life events), as well as the underlying dyadic processes in couples (e.g., support provision, conflict management) in order to understand and predict variations in marital outcomes of couples facing pediatric cancer (e.g., Vulnerability-stress-adaptation model [69]). In addition, matching the unit of interest with the unit of measurement requires research involving both partners of the couple and taking into account their interdependence. This practice was lacking in most of the included studies but would allow for a more detailed analysis of couple functioning. One way to do this, as well as to go beyond the well-known disadvantages of global self-reports, is the use of observational methods or diary methods. Lastly, only about half of the included studies were quantitative. More quantitative research utilizing longitudinal designs with large, representative samples would benefit this field.

\section{Clinical implications}

Based upon our review, we can conclude that problems within the couple subsystem only seem to occur for a subset of families, and that most couples adapt well after a pediatric cancer diagnosis. Although we cannot be sure, couples experiencing those problems likely comprise those with pre-existing problems as well as those having difficulty specifically because of the stressor of childhood cancer. 
Because these difficulties in the couple relationship may seem secondary to the more pressing need of ensuring adequate cancer and psychosocial care for the child, such issues may be overlooked by psychosocial providers in oncology or even seen as outside their purview of care. In addition, these problems may also be downplayed by the couple themselves (put on a back burner), as they as well primary focus on their sick child and his/her treatment process. However, as these problems might negatively impact the adjustment of the child and his/her treatment, it is important to screen and remedy those problems, taking into account evidence based standards for psychosocial care in pediatric oncology [71]. For example, one can imagine that couples with different coping styles might experience elevated distress, anxiety or depression [45,72], which may be linked -in turn- with child distress and/or behavior problems. Studies assessing the direct influence of marital quality on psychosocial outcomes in children with cancer, however, are missing [73].

Interventions aimed at dealing with couple problems that get in the way of cancer care or hamper the adjustment of the child and family would ideally involve both members of the couple. However, efforts to provide such intervention formats may be difficult to achieve in practice [74] and practitioners may need to rely on technology (e.g., telemedicine) to conduct conjoint sessions or work with members of the couple individually. In addition, the goals of such interventions may need to focus on finding ways for the couple to work together effectively to meet the needs of their child and family during cancer care, rather than making progress on long-standing difficulties within the couple. Once the couple is working together more effectively and capably managing the stress of cancer, then they should receive referral to community providers to address relational issues outside of cancer. 


\section{References}

1. Cancer Research UK. One-, Five- and Ten-Year Actuarial Survival. Cancer Research UK 2013. Retrieved January 15, 2016, from the world wide web: http://www.cancerresearchuk.org/sites/default/files/cstream-node/child_surv_gb_1510yr.pdf

2. Kestler SA, LoBiondo-Wood G. Review of symptom experiences in children and adolescents with cancer. Cancer Nursing 2012; 35(2): E31-E49. doi:10.1097/NCC.0b013e3182207a2a

3. Alderfer MA, Long KA, Lown EA, Marsland AL, Ostrowski NL, Hock, JM, Ewing, LJ. Psychosocial adjustment of siblings of children with cancer: a systematic review. PsychoOncology 2010; 19(8): 789-805. doi: 10.1002/pon.1638

4. Kazak AE, Alderfer M, Rourke MT, Simms S, Streisand R, Grossman JR. Posttraumatic stress disorder (PTSD) and posttraumatic stress symptoms (PTSS) in families of adolescent childhood cancer survivors. Journal of Pediatric Psychology 2004; 29(3): 211219. doi: 10.1093/jpepsy/jsh022

5. Alderfer MA, Kazak AE. Family issues when a child is on treatment for cancer. In Comprehensive Handbook of Childhood Cancer and Sickle Cell Disease: A Biopsychosocial Approach, Brown R. (ed.). Oxford University Press: New York, 2006; 53-74.

6. Stam H, Grootenhuis MA, Last BF. Social and emotional adjustment in young survivors of childhood cancer. Supportive Care in Cancer 2001; 9: 489-513. doi: 10.1007/s005200100271

7. Grootenhuis MA, Last BF. Adjustment and coping by parents of children with cancer: A review. Supportive Care Cancer 1997; 5: 466-484. doi: 10.1007/s005200050116 
8. Patino-Fernandez AM, Pai A, Alderfer MA, Hwang WT, Reilly A, Kazak AE. Acute stress in parents of children newly diagnosed with cancer. Pediatric Blood \& Cancer 2008; 50: 289-292. doi: 10.1002/pbc.21262

9. Houtzager B, Grootenhuis M, Last B. Adjustment of siblings to childhood cancer: a literature review. Supportive Care in Cancer 1999; 7: 302-320. doi: 10.1007/s005200050268

10. Van Schoors M, Caes L, Verhofstadt LL, Goubert L, Alderfer MA. (2015). Systematic Review: Family Resilience After Pediatric Cancer Diagnosis. Journal of Pediatric Psychology 2015; 40(9): 856-868. doi: 10.1093/jpepsy/jsv055

11. Minuchin S. Families and family therapy. Cambridge, HA: Harvard University Press; 1974.

12. Bronfenbrenner, U. Toward an experimental ecology of human development. American Psychologist 1977; 32(7): 513-531. doi: 10.1037/0003-066X.32.7.513

13. McCubbin HI, Patterson JM. The family stress process: The double ABCX model of adjustment and adaptation. Marriage \& Family Review 1983; 6(1-2): 7-37. doi: 10.1300/J002v06n01_02

14. Patterson, JM. Families experiencing stress: I. The Family Adjustment and Adaptation Response Model: II. Applying the FAAR Model to health-related issues for intervention and research. Family Systems Medicine 1988; 6(2): 202-237. doi: 10.1037/h0089739

15. Bradbury TN, Karney BR. Intimate relationships. W. W. Norton \& Company: New York, 2014.

16. Spanier GB, Lewis RA. Marital quality: A review of the seventies. Journal of Marriage and the Family 1980; 42(4): 825-839. doi: jstor.org/stable/351827

17. Schaer M, Bodenmann, G. Stress and strain due to development processes in families: coping strategies as in the example of dyadic coping. In Family Diversity: Collection of the 3rd European 
Congress of Family Science, Kapella O, Pfeiffer RC, Rupp M, Scheider NF (eds.). Barbara Budrich Publishers: Leverkusen-Opladen, 2010; 371-378

18. Hildenbrand AK, Clawson K.J, Alderfer MA, Marsac ML. (2011). Coping With Pediatric Cancer: Strategies Employed by Children and Their Parents to Manage Cancer-Related Stressors During Treatment. Journal of Pediatric Oncology Nursing 2011; 28(6): 344-354. doi:10.1177/1043454211430823

19. Greenberg HS, Meadows AT. Psychosocial impact of cancer survival on school-age children and their parents. Journal of Psychosocial Oncology 1992; 9(4); 43-56. doi: 10.1300/J077v09n04_03

20. Van Schoors M, Caes L, Verhofstadt LL, Goubert L, Alderfer MA.Systematic Review: Family Resilience After Pediatric Cancer Diagnosis. J Pediatr Psychol. 2015;40(9):856868. doi: 10.1093/jpepsy/jsv055

21. Van Schoors M, Caes L, Knoble NB, Goubert L, Verhofstadt LL, Alderfer MA. Family functioning and child adjustment after pediatric cancer diagnosis:A systematic review and meta - analysis. J Pediatr Psychol. In press

22. Eiser C, Hill J, Vance Y. Examining the psychological consequences of surviving childhood cancer: Systematic review as a research method in pediatric psychology. Journal of Pediatric Psychology 2000; 25: 449-460. doi: 10.1093/jpepsy/ 25.6.449

23. Higgins JPT, Green S. Cochrane (Eds.) Cochrane handbook for systematic reviews of interventions version 5.1.0. The Cochrane Collaboration, 2011. Available from www.handbook.cochrane.org

24. Davis, KE. Closeness. In Encyclopedia of Human Relationships, Reis HT, Sprecher S. (eds.). Sage Publications: Thousand Oaks, 2009; 215-219. 
25. Beltrao MR, Vasconcelos MG, Pontes CM, Albuquerque MC. Childhood cancer: maternal perceptions and strategies for coping with diagnosis. Journal de Pediatra (Rio J) 2007; 83(6): 562566. doi:10.2223/JPED.1723

26. Brody AC, Simmons LA. Family resiliency during childhood cancer: The father's perspective. Journal of Pediatric Oncology Nursing 2007; 24(3): 152-165. doi: $10.1177 / 1043454206298844$

27. Enskar K, Carlsson M, Golsater M, Hamrin E, Kreuger A. Parental reports of changes and challenges that result from parenting a child with cancer. Journal of Pediatric Oncololgy Nursing 1997; 14(3):156-163. doi: 10.1016/S1043-4542(97)90051-5

28. Khoury MN, Huijer HA, Doumit MA. Lebanese parents' experiences with a child with cancer. European Journal of Oncology Nursing 2013; 17(1): 16-21. doi: 10.1016/j.ejon.2012.02.005

29. Patistea E, Makrodimitri P, Panteli, V. Greek parents' reactions, difficulties and resources in childhood leukaemia at the time of diagnosis. European Journal of Cancer Care 2000; 9(2), 86-96. doi: 10.1046/j.1365-2354.2000.00204.x

30. Tremolada M, Bonichini S, Aloisio D, Schiavo S, Carli M, Pillon M. Post-traumatic stress symptoms among mothers of children with leukemia undergoing treatment: a longitudinal study. Psychooncology 2013; 22(6): 1266-1272. doi: 10.1002/pon.3132

31. Fincham, FD. Closeness. In Encyclopedia of Human Relationships, Reis HT, Sprecher S. (eds.). Sage Publications: Thousand Oaks, 2009; 298-303.

32. Wills, BS. The experiences of Hong Kong Chinese parents of children with acute lymphocytic leukemia. Journal of Pediatric Nursing 1999; 14(4): 231-238. doi: $10.1016 / \mathrm{S} 0882-5963(99) 80017-7$ 
33. Patterson JM, Holm KE, Gurney JG. The impact of childhood cancer on the family: A qualitative analysis of strains, resources, and coping behaviors. Psycho-Oncology 2004; 13(6): 390-407. doi: 10.1002/pon.761

34. Lavee Y, Mey - Dan M. Patterns of change in marital relationships among parents of children with cancer. Health Soc Work. 2003;28(4):255-263.doi: 10.1093/hsw/28.4.255.

35. Lahteenmaki PM, Sjoblom J, Korhonen T, Salmi TT. The life situation of parents over the first year after their child's cancer diagnosis. Acta Paediatrica 2004; 93(12): 1654-1660. doi: $10.1080 / 08035250410033033$

36. McGrath, P. Identifying support issues of parents of children with leukemia. Cancer Practice 2001; 9(4): 198-205. doi: 10.1046/j.1523-5394.2001.94008.x

37. Walsh, E. Strengthening Family Resilience. Guilford Press: New York, 1998.

38. Barbarin OA, Hughes D, Chesler MA. Stress, coping, and marital functioning among parents of children with cancer. J Marriage Fam. 1985;47(2):473 - 480. http://www.jstor.org/stable/352146

39. Wills, BS. Coping with a child with acute lymphocytic leukemia: the experiences of Chinese fathers in Hong Kong. Cancer Nursing 2009; 32(2): E8-E14. doi: 10.1097/01.NCC.0000339260.98818.9c

40. Mercer M, Ritchie JA. Tag team parenting of children with cancer. Journal of Pediatric Nursing 1997; 12(6): 331-341. doi: 10.1016/S0882-5963(97)80059-0

41. Morrow GR, Hoagland AC, Morse IP. Sources of support perceived by parents of children with cancer: implications for counselling. Patient Counseling and Health Education 1982; 4(1): 36-40. doi: 10.1016/S0738-3991(82)80033-5 
42. Penn A, Lowis SP, Stevens MCG, Hunt LP, Shortman RI, McCarter RJ, Pauldhas D, Curran AL, Sharples PM. Family, Demographic and Illness-Related Determinants of HRQL in Children With Brain Tumours in the First Year After Diagnosis. Pediatric Blood \& Cancer 2009; 53(6): 1092-1099. doi: 10.1002/pbc.22157

43. Olson, DH. Circumplex model of marital and family systems. Journal of Family Therapy 2000; 22:144-167. doi: 10.1111/1467-6427.00144

44. Wijnberg-Williams BJ, Van de Wiel HBM, Kamps W, Hoekstra-Weebers JEHM. Effects of communication styles on marital satisfaction and distress of parents of pediatric cancer patients: A prospective longitudinal study. Psycho-Oncology 2015; 24(1): 106-112. doi: 10.1002/pon.3617

45. Manne S, DuHamel K, Winkel G, Ostroff J, Parsons S, Martini R, Williams SE, Mee L, Austin J, Redd WH. Perceived partner critical and avoidant behaviors as predictors of anxious and depressive symptoms among mothers of children undergoing hemopaietic stem cell transplantation. Journal of Consulting and Clinical Psychology 2003; 71(6): 1076-1083. doi: 10.1037/0022-006x.71.6.1076

46. Canary DJ, Emmers-Sommer TM, Faulkner, S. Sex and gender differences in personal relations. The Guildford Press: New York, 1997.

47. Ferrell BR, Rhiner M, Shapiro B, Dierkes M. The experience of pediatric cancer pain, Part I: Impact of pain on the family. Journal of Pediatric Nursing 1994; 9(6): 368-379.

48. Lawrence E, Barry RA, Langer A, Brock RL. Marital Satisfaction, Assessment of. In Encyclopedia of Human Relationships, Reis HT, Sprecher S. (Eds.). Sage Publications: Thousand Oaks, 2009; 1028-1030. 
49. Hoekstra-Weebers J, Jaspers JPC, Kamps WA, Klip EC. Marital dissatisfaction, psychological distress, and the coping of parents of pediatric cancer patients. Journal of Marriage and the Family 1998; 60(4): 1012-1021. doi: 10.2307/353642

50. Yeh, $\mathrm{CH}$. Gender differences of parental distress in children with cancer. Journal of Advanced Nursing 2002; 38(6): 598-606. doi: 10.1046/j.1365-2648.2000.02227.x

51. Brown RT, Kaslow NJ, Hazzard AP, Madan-Swain A, Sexson, SB, Lambert R, Baldwin K. Psychiatric and family functioning in children with leukemia and their parents. Journal of American Academy of Child and Adolescent Psychiatry 1992; 31(3): 495-502. doi:10.1097/00004583-199205000-00017

52. Fife B, Norton J, Groom G. The family's adaptation to childhood leukemia. Social Science \& Medicin 1987; 24(2): 159-168. doi: 10.1016/0277-9536(87)90248-6

53. Shapiro J, Shumaker S. Differences in emotional well-being and communication styles between mothers and fathers of pediatric cancer patients. Journal of Psychosocial Oncology 1987; 5(3): 121-131. doi: 10.1300/J077v05n03_06

54. Fletcher PC, Clarke J. When Your Child Has Cancer: A Discussion of Factors That Affect Mothers' Abilities to Cope. Journal of Psychosocial Oncology 2003; 21(3); 81-99. doi: 10.1300/J077v21n03_05

55. Lansky SB, Cairns NU, Hassanein R, Wehr J, Lowman JT. Childhood cancer: parental discord and divorce. Pediatrics. 1978;62(2):184-188.

56. Dahlquist LM, Czyzewski DI, Copeland KG, Jones CL, Taub E, Vaughan JK. Parents of children Newly-Diagnosed with Cancer: Anxiety, Coping and Marital Distress. Journal of Pediatric Psychology 1993; 18(3): 365-376. doi: 10.1093/jpepsy/18.3.365 
57. Dahlquist LM, Czyzewski DI, Jones CL. Parents of children with cancer: A longitudinal study of emotional distress, coping style, and marital adjustment two and twenty months after diagnosis. Journal of Pediatric Psychology 1996; 21(4): 541-554. doi: 10.1093/jpepsy/21.4.541

58. Cornman, BJ. Childhood cancer: differential effects on the family members. Oncology Nursing Forum 1993; 20(10): 1559-1566.

59. Lavee, Y. Correlates of change in marital relationships under stress: The case of childhood cancer. Families in Society-the Journal of Contemporary Social Services 2005; 86(1): 112-120. doi: 10.1606/1044-3894.1883

60. Wittrock DA, Larson LS, Sandgren AK. When a child is diagnosed with cancer: II. Parental coping, psychological adjustment, and relationships with medical personnel. Journal of Psychosocial Oncology 1994; 12(3): 17-32. doi: 10.1300/J077V12N03_02

61. Cook WL, Kenny DA. The Actor-Partner Interdependence Model: A model of bidirectional effects in developmental studies. International Journal of Behavioral Development 2005; 29(2): 101-109. doi: 10.1080/01650250444000405

62. Weber, J. Individual and Family Stress and Crises. Sage, Inc.: Thousand Oaks, 2011. 63. Alderfer MA, Navsaria N, Kazak AE. Family functioning and Posttraumatic Stress Disorder in adolescent survivors of childhood cancer. Journal of Family Psycholology 2009; 23: 717-725. doi: 10.1037/a0015996

64. Peterson CC, Cousino MK, Donohue JE, Schmidt ML, Gurney JG.Discordant parent reports of family functioning following childhood neuroblastoma. Journal of Psychosocial Oncology 2012; 30: 503-518. doi: 10.1080/07347332.2012.703766 
65. Schwarz N, Groves RM, Schuman H. Survey methods. In The Handbook of Social Psychology (4th ed., vol. 2), Gilbert DT, Fiske S, Lindzey G. (Eds.). McGraw-Hill: Boston, 1998; 143-179

66. Hilliard ME, Harris MA, Weissberg-Benchell J. Diabetes resilience: a model of risk and protection in type 1 diabetes. Current diabetes reports 2012; 12(6): 739-748. doi: $10.1007 / \mathrm{s} 11892-012-0314-3$

67. Alderfer MA, Stanley C. Health and illness in the context of the family. In Handbook of Health Psychology, Second Edition, Baum A, Revenson TA, Singer JE (Eds.). Taylor \& Francis: London, 2012; 493-516.

68. Berger LM, Bzostek SH. Young adults' roles as partners and parents in the context of family complexity. The ANNALS of the American Academy of Political and Social Science 2014; 654(1): 87-109. doi: 10.1177/0002716214527729

69. Karney BR, Bradbury TN. The longitudinal course of marital quality and stability: A review of theory, methods, and research. Psychological bulletin 1995; 118(1): 3-34. doi:10.1037/00332909.118.1.3

70. Kluwer, ES. From partnership to parenthood: A review of marital change across the transition to parenthood. Journal of Family Theory \& Review 2010; 2(2): 105-125. doi: $10.1111 / \mathrm{j} .17562589 .2010 .00045 . \mathrm{x}$

71. Wiener LK, Anne E, Robert NB, Patenaude AF, Kupst MJ. Standards for the psychosocial care of children with cancer and their families: An introduction to the special Issue. Pediatric Blood \& Cancer 2015; 62: S419-S424. doi: 10.1002/pbc.25675

72. Hoekstra-Weebers J, Jaspers JPC, Kamps WA, Klip EC (1998). Gender differences in psychological adaptation and coping in parents of pediatric cancer patients. Psycho- 
Oncology 1998; 7(1): 26-36. doi: 10.1002/(sici)1099-1611(199801/02)7:1<26::aidpon315>3.0.co;2-0

73. Long KA, Marsland AL. Family adjustment to childhood cancer: A systematic review. Clinical Child and Family Psychology Review 2011; 14:57-88. DOI 10.1007/s10567-0100082-z

74. Stehl ML, Kazak AE, Alderfer, MA, Rodriguez A, Hwang WT, Pai ALH, Boeving A, Reilly A. Conducting a randomized clinical trial of an psychological intervention for parents/caregivers of children with cancer shortly after diagnosis. Journal of Pediatric Psychology 2009: 34(8): 803-816. doi: 10.1093/jpepsy/jsn130 